\title{
THE RISK ASSESSMENT AND MANAGEMENT OF PREMATURE SCREEN-OUT DURING HYDRAULIC FRACTURING BASED ON THE BAYESIAN BELIEF NETWORK MODEL
}

\author{
ENRICO ZIO ${ }^{1,2,3}$, MARYAM MUSTAFAYEVA $^{4}$ and ANDREA MONTANARO 5
}

${ }^{1}$ Centre de Recherche sur les Risques et les Crises (CRC), MINES ParisTech/PSL Université Paris, Sophia Antipolis, France.

${ }^{2}$ Department of Energy, Politecnico di Milano, Milan, Italy.

${ }^{3}$ Eminent Scholar at the Department of Nuclear Engineering, Kyung Hee University, Seoul, South Korea

E-mail: enrico.zio@polimi.it

\author{
${ }^{4}$ Department of Management, Economics and Industrial Engineering, Politecnico di Milano, \\ Milan, Italy. \\ ${ }^{5}$ Kwantis, Milan, Italy.
}

Hydraulic fracturing is a widespread well completion technique for Oil and Gas production enhancement in both conventional and unconventional reservoirs. However, it is exposed to various risks, which can result in a most adverse consequence - the premature screen-out, which is a condition that occurs due to the proppant bridging across the perforations or similar restricted flow areas.

The goal of this work is to propose a novel and complete approach that makes it possible to predict the risk of screen-out occurrence, identify the riskiest scenarios and determine the best risk reduction strategies. The premature screen-out problem is addressed within a Risk Management and Control Process. The qualitative assessment of the early screen-out risk has been carried out by the Features, Events and Processes (FEP) Analysis, and the quantitative assessment has been implemented with a Bayesian Belief Network (BBN). The input values of the BBN model, i.e. the probabilities of the model's variables, have been evaluated using various expert elicitation methods due to shortfall in data, and the log-likelihood method has turned out to provide the best results.

The BBN model has been examined by uncertainty and sensitivity analyses. The results of the uncertainty analysis have shown the robustness the BBN model in giving adequate evaluations. The global sensitivity analysis of the BBN model has been performed by the Sobol's variance decomposition method. The probabilities of the most influential input parameters as identified by the sensitivity analysis have been re-evaluated in order to reduce the output uncertainty. As a result, the probability of screen-out has decreased.

Finally, risk mitigation plans have been formulated using risk importance measures to determine the riskiest scenarios and cost-benefit analysis to compare the effects of different risk reduction actions.

The main novel contributions of this work lie in the use of the BBN framework for the assessment of the risk of early screen-out, in the determination of the most influential variables by the Sobol's first-order and total-effects sensitivity indices and in the identification of optimal risk reduction strategies by the use of risk importance measures and cost-benefit analysis. 